\title{
Genome-wide identification and characterization of tRNA- derived RNA fragments in land plants
}

\author{
Cristiane S. Alves $^{1,2} \cdot$ Renato Vicentini $^{3}$ - Gustavo T. Duarte ${ }^{4}$ Vitor F. Pinoti ${ }^{1}$. \\ Michel Vincentz ${ }^{4} \cdot$ Fabio T. S. Nogueira ${ }^{2}$ (D)
}

Received: 20 May 2016 / Accepted: 19 September 2016 / Published online: 28 September 2016

(C) Springer Science+Business Media Dordrecht 2016

\begin{abstract}
Key message The manuscript by Alves et al. entitled "Genome-wide identification and characterization of $t R \boldsymbol{N} A$-derived $R \boldsymbol{R}$ A fragments in land plants" describes the identification and characterization of tRNAderived sRNA fragments in plants. By combining bioinformatic analysis and genetic and molecular approaches, we show that tRF biogenesis does not rely on canonical microRNA/siRNA processing machinery (i.e., independent of DICER-LIKE proteins). Moreover, we provide evidences that the Arabidopsis S-like Ribonuclease 1 (RNS1) might be involved in the biogenesis of tRFs. Detailed analyses showed that plant tRFs are sorted into different types of ARGONAUTE proteins and that they have potential target candidate genes. Our work advances the understanding
\end{abstract}

Electronic supplementary material The online version of this article (doi:10.1007/s11103-016-0545-9) contains supplementary material, which is available to authorized users.

Fabio T. S. Nogueira

ftsnogue@usp.br

Cristiane S. Alves

cris_desantis@icloud.com

Renato Vicentini

shinapes@unicamp.br

Gustavo T. Duarte

duarte.gst@gmail.com

Vitor F. Pinoti

vitorpinoti@yahoo.com.br

Michel Vincentz

mgavince@unicamp.br

1 Departamento de Genetica, Instituto de Biociencias, Universidade Estadual Paulista (UNESP), Distrito de Rubião Jr., s/n, Botucatu, SP 18618-970, Brazil of the tRF biology in plants by providing evidences that plant and animal tRFs shared common features and raising the hypothesis that an interplay between tRFs and other sRNAs might be important to fine-tune gene expression and protein biosynthesis in plant cells.

Abstract Small RNA (sRNA) fragments derived from tRNAs (3'-loop, 5'-loop, anti-codon loop), named tRFs, have been reported in several organisms, including humans and plants. Although they may interfere with gene expression, their biogenesis and biological functions in plants remain poorly understood. Here, we capitalized on small RNA sequencing data from distinct species such as Arabidopsis thaliana, Oryza sativa, and Physcomitrella patens to examine the diversity of plant tRFs and provide insight into their properties. In silico analyzes of 19 to 25-nt tRFs derived from 5' (tRF-5s) and 3'CCA (tRF-3s) tRNA loops in these three evolutionary distant species showed that they are conserved and their abundance did not correlate with the number of genomic copies of the parental tRNAs. Moreover,

2 Laboratorio de Genetica Molecular do Desenvolvimento Vegetal, Departamento de Ciencias Biologicas, ESALQ/USP, Avenida Pádua Dias s/n, 11, Piracicaba, SP 13418-900, Brazil

3 Laboratorio de Bioinformatica e Biologia de Sistemas, Departamento de Genetica, Evoluçao e Bioagentes, Universidade Estadual de Campinas (Unicamp), Campinas, SP, Brazil

4 Centro de Biologia Molecular e Engenharia Genetica (CBMEG), Universidade Estadual de Campinas (Unicamp), Campinas, SP, Brazil 
tRF-5 is the most abundant variant in all three species. In silico and in vivo expression analyses unraveled differential accumulation of tRFs in Arabidopsis tissues/organs, suggesting that they are not byproducts of tRNA degradation. We also verified that the biogenesis of most Arabidopsis 19-25 nt tRF-5s and tRF-3s is not primarily dependent on DICER-LIKE proteins, though they seem to be associated with ARGONAUTE proteins and have few potential targets. Finally, we provide evidence that Arabidopsis ribonuclease RNS1 might be involved in the processing and/or degradation of tRFs. Our data support the notion that an interplay between tRFs and other sRNAs might be important to fine tune gene expression and protein biosynthesis in plant cells.

Keywords Small RNA - tRNA-derived RNA fragment · tRF $\cdot$ Arabidopsis thaliana $\cdot$ High throughput data

\section{Introduction}

Transfer RNAs (tRNAs) are an ancient form of non-coding RNA (ncRNA) that provides a connection between transcription and translation in most organisms. Each eukaryotic genome has at least 32 distinct families of tRNAs to decode the 20 amino acids in a proper way. tRNA biogenesis is a complex process that involves several enzymes, distinct cell localization for each step and usually differs among yeast, plants and vertebrates. Apparently, tRNA biogenesis pathway in a particular species relies on the evolution of the processing enzymes found in each species (for review, see Hopper and Shaheen 2008). Interestingly, tRNAs can assume additional biological and cellular tasks such as an unexpected role in inhibiting apoptosis by binding cytochrome $c$ and consequently blocking the caspase activation in HeLa cells (Mei et al. 2010). Furthermore, they can be used as primers for viral reverse transcription, while uncharged tRNAs can act as stress sensors and as regulators of gene expression (for review see Phizicky and Hopper 2010). Recently new findings reveal that tRNAs may perform non-canonical, extra-translational functions that may be linked to human disease (Parisien et al. 2013).

Advances in high-throughput sequencing techniques allowed the identification of small RNAs (sRNAs) derived from various cellular non-coding RNAs, including tRNAs. The most known and well-studied sRNAs are typically 19-25 nt in length and are classified into microRNAs (miRNAs), endogenous small interfering RNAs (endo-siRNAs), trans-acting siRNAs (ta-siRNAs), and Piwi-interacting small RNAs (piRNAs) (Nogueira et al. 2006; Farazi et al. 2008). Recent studies have demonstrated the existence of novel classes of sRNAs and that RNA interference may be more ubiquitous than previously appreciated (Tuck and Tollervey 2011; Olovnikov et al. 2013). For example, tRNA halves (stress-induced small RNAs or short tiRNAs), deriving from both the $5^{\prime}$ and $3^{\prime}$ halves of the tRNA molecule, are produced by cleavage in the anticodon loop (Emara et al. 2010). Interestingly, tRNA halves can be generated in response to oxidative stress in several eukaryotic species, including plants (Thompson et al. 2008).

In addition to tRNA halves, small RNAs deriving from mature tRNAs, collectively termed tRNA-derived RNA fragments (tRFs), have been firstly discovered in HeLa cells (Lee et al. 2009) and further in different species and cell types ( $\mathrm{Li}$ et al. 2012; Haussecker et al. 2010; Hsieh et al. 2009; Cole et al. 2009; Cai et al. 2013; Sobala et al. 2013). Two main types of tRFs can be originated according to the processing of the parental tRNA: the first type derives from the $5^{\prime}$ end (tRF-5s), while the second derives from the $3^{\prime}$ end, harboring the added CCA sequence (3' CCA tRFs or tRF-3s) (Gebetsberger and Polacek 2013; Fu et al. 2015). Although some early reports have implicated DICERs in the generation of particular tRFs, more recent studies suggest that in human cells DICER is dispensable for the generation of most tRF-5s and tRF-3s (Li et al 2012; Kumar et al. 2014). Indeed, most tRFs are essentially restricted to the cytoplasm and some have been shown to be produced by the action of other endonucleases, such as Angiogenin (Yamasaki et al. 2009). Therefore, it is possible that a combination of ribonucleases is necessary for generating different types of tRFs (Sobala and Hutvagner 2013).

While cellular functions have been assigned for tRNA halves (Thompson et al. 2008; Yamasaki et al. 2009; Emara et al. 2010), functions of the tRNA-derived RNA fragments proposed so far do not yet allow drawing a clear picture of their RNA biology. Cellular and molecular functions of tRFs may be mediated by their association with Argonaute (AGO) proteins (Cole et al. 2009; Haussecker et al. 2010; Li et al. 2012; Kumar et al. 2014) and, at least in some instances, may involve the inhibition of protein translation and downregulation of target genes by transcript cleavage, resembling miRNA/siRNA biological functions (Li et al. 2012; Sobala and Hutvagner 2013). Another interesting hypothesis is that tRFs may compete for AGO association with other cellular sRNAs and thus they might act as global regulators of RNA-mediated silencing (Haussecker et al. 2010). These findings suggest that tRNA-derived sRNA molecules are, in some cell types and biological contexts, used for broad control of gene expression.

Studies evaluating the expression, biogenesis and possible biological functions of tRFs have been mainly performed in animal cells, while in plants it remains poorly explored. In Arabidopsis thaliana, the tRF-5s and tRF-3s of $\mathrm{Asp}^{\mathrm{GTC}}$ and Gly ${ }^{\mathrm{TCC}}$ tRNAs were found to be highly expressed in root tissues grown under conditions of phosphate deprivation (Hsieh et al. 2009). In barley, the His GTG tRF-5 was the most abundant sRNA molecule identified in 
small RNA populations under phosphorous-deficient conditions (Hackenberg et al. 2012), while Ala ${ }^{A G C}$ and Pro ${ }^{\text {CGG }}$ tRF-5s showed differential expression in rice callus and leaves (Chen et al. 2011). Additionally, some tRF-5s were shown to be associated with Arabidopsis AGO proteins (Loss-Morais et al. 2013). In this study, we exploited publicly small RNA sequencing data from Arabidopsis, rice and moss to comprehensively examine the diversity, expression and possible biogenesis of tRFs in plants. We showed that tRF-5s and tRF-3s are conserved across plant kingdom and their accumulation is not correlated with parental tRNA gene copy number. Although they incorporate into AGOs, most plant tRF-5s and tRF-3s are not generated by the canonical miRNA biogenesis pathway. We also provided evidences that both types of tRFs accumulated in response to oxidative stress and that Arabidopsis RNA-associated enzyme RNS1 might be involved in the biogenesis and/or accumulation of some tRFs.

\section{Materials and methods}

\section{Dataset and bioinformatics}

The data analyzed in this study were downloaded from GEO database (Barrett et al. 2013). We considered only those sets of high throughput sequencing data in which small RNAs of 14 to 36 bases long were size selected and then sequenced. Datasets of different genotypes and experiments (Online Resource 1) were mined for tRF identification. By performing BLAST alignment (minimal identity $=100$, tile size $=7$, and minimal score $=0$ ), we considered only those alignments where the query sequence (sRNA) had a perfect match against tRNAs from Arabidopsis thaliana $(\mathrm{n}=639)$, Oryza sativa $(\mathrm{n}=764)$ and Physcomitrella paten $\mathrm{s}(\mathrm{n}=426)$, obtained from GtRNAdb (Chan and Lowe 2009). Given that 'CCA' is added at the 3 ' end of tRNA by tRNA nucleotidyltransferase during maturation of tRNA (Xiong and Steitz 2006), we allowed exception for mapping sRNAs to the $3^{\prime}$ ends of tRNAs in the GtRNAdb allowing a terminal mismatch of $\leq 3$ bases.

tRF expression profiles were estimated normalizing the amount of reads of each sRNA by the total sRNA library sequence size, with a minimal of 10 per 1 million of reads. tRFs were considered differentially expressed when Fold Change $(\mathrm{FC}) \leq$ or $\geq 1$, where FCLOG $2=\mathrm{tRF}$ experiment or mutant/tRF control experiment or WT.

Potential tRF target candidates were mined by using psRNATarget (Dai and Zhao 2011). Parallel analysis of RNA end (PARE) signatures for possible tRF target candidates were retrieved from libraries that contain $3^{\prime}$ cleavage products of mRNAs. Such libraries were generated by Dr. Meyers Lab at the Donald Danforth Plant Science Center (https://mpss.danforthcenter.org). PARE signatures were mapped to the respective transcripts as described (German et al. 2008). Initially, we matched sequences flanking potential cleavage sites of RNA cleavage products (PARE signatures) back to their corresponding mRNA sequences. Such mRNA sequences corresponded to experimentally confirmed miRNA targets, which were identified by psRNATarget. We used similar approach to identify PARE signatures for putative tRF targets.

\section{Plant material and growth conditions}

Arabidopsis thaliana plants (ecotype Columbia-0 or Col0 ) were grown at $21^{\circ} \mathrm{C}$ (day) $/ 19^{\circ} \mathrm{C}$ (night) under long-day conditions (16 h light/8 h dark). Mutants hyll-2 (Vazquez et al. 2004), $d c l 1-11$ (Zhang et al. 2008), dcl2-1 and $d c l 3$ 1 (Xie et al. 2004), dcl4-2 (Xie et al. 2005) were previously described. The rns $1-1$ mutant in Col-0 background (salk_087165C) was obtained from TAIR stock (https:// www.Arabidopsis.org/). All mutants were confirmed by PCR genotyping.

\section{Oxidative stress treatment}

Arabidopsis seeds were surface sterilized and stratified for $48 \mathrm{~h}$ at $4{ }^{\circ} \mathrm{C}$ them transferred to a growth chamber. Seedlings were growth on erlenmeyers containing half strength modified Murashige and Skoog ( $1 / 2 \mathrm{MS})$ medium with vitamins (PhytoTechnology Laboratories ${ }^{\circledR}$ ) and $1.5 \%$ sucrose. Seedlings were growth under constantly light at $19-22^{\circ} \mathrm{C}$. After 9 days, $10 \mathrm{mM} \mathrm{H}_{2} \mathrm{O}_{2}$ was added to $1 / 2 \mathrm{MS}$ medium (Thompson et al. 2008). For control plants, $1 / 2 \mathrm{MS}$ was used instead $\mathrm{H}_{2} \mathrm{O}_{2}$. After $16 \mathrm{~h}$, both control and treated seedlings were harvested and quickly frozen in liquid nitrogen. The experiment was repeated twice.

\section{Tissue/organ collection, RNA isolation, and real-time RT-PCR analysis}

For tissue/organ and rns1-1 mutant expression analyses, rosette leaves and flower buds were collected from 60-dayold plants. Tissues of 15-day-old seedlings from Col-0, dcl1-11, dcl2-1, dcl3-1, dcl4-2, and hyl1-2 were collected and for each biological sample, a pool of 15 plants was used. The plant material was grounded under liquid nitrogen and total RNA extracted with TRIzol ${ }^{\circledR}$ Reagent (Ambion) according to manufacturer instructions. Total RNA was treated with Turbo DNA-free ${ }^{\mathrm{TM}}$ (Ambion). To detected and quantify tRF transcripts (tRF-5 Arg ${ }^{\mathrm{TCG}}$ from tRNA-Arg ${ }^{\mathrm{TCG}}$; tRF-5 Arg ${ }^{\mathrm{CCT}}$ from tRNA-Arg ${ }^{\mathrm{CCT}}$; tRF-3 $\mathrm{Tyr}^{\mathrm{GTA}}$ from tRNA-Tyr ${ }^{G T A}$; and tRF-3 Ser ${ }^{G G A}$ from tRNA-Ser ${ }^{G G A}$ ), stem-loop RT-qPCR was performed according to Chen and coworkers (2011). The reactions were carried out using 
Go-Taq ${ }^{\circledR}$ qPCR Master Mix (Promega), following the manufacturer instructions. Applied StepOne ${ }^{\mathrm{TM}}$ Real-Time PCR System was used to perform the reactions. For all analyses, Actin2 or ACT2 (AT3G18780) was used as an endogenous gene. All reactions were run in triplicate with three biological samples. Differences in gene expression were evaluated by the Delta-Delta Ct method (Livak and Schmittgen 2001) and considered significant for $p$ values of $<0.05$ according to two-tailed Student's $t$ test. PCR specificity was examined by melting curve analysis from 65 to $95^{\circ} \mathrm{C}$, and the products were checked in a $2.5 \%$ agarose gel. The amplicons of $\sim 60$ bp were cloned into pGEM $^{\circledR}$-T Easy Vector (Promega) and tRF identity was confirmed by sequencing two clones of each corresponding tRF. All primers are described in Online Resource 2.

\section{Results and discussion}

\section{Overall characterization of tRFs in evolutionarily distant plant species}

Using publicly available databases of small RNA libraries from distinct evolutionary plant species (Online Resource 1), we selected reads from 19 to $25 \mathrm{nt}$, previously reported to be the most abundant $5^{\prime}$ and $3^{\prime} \mathrm{CCA}$ tRNA-derived RNA fragments (tRF-5s and tRF-3s, respectively) in different species (Lee et al. 2009; Cai et al. 2013). Next, we mapped their origin (i.e., parental tRNA), considering only reads that fully mapped to the respective mature tRNAs. We considered representative tRF-5s and tRF-3s those presenting at least 10 reads per million (RPM). For Arabidopsis and rice, we selected tRF-5s and tRF-3s which are conserved between the two species. In terms of evolution, moss can be considered as a basal species for land plants, from which arose around 500 millions of years ago (Kenrick and Crane 1997; Prigge and Bezanilla 2010). Therefore, we decided to analyze all $P$. patens tRF-5s and tRF-3s (conserved and non-conserved in Arabidopsis and rice), which fully mapped to their respective parental tRNAs (see "Materials and methods").

Firstly, we asked whether there was a correlation between parental tRNA loci number and tRF abundance by estimating the total number of tRNA loci from each anticodon and the number of tRFs that mapped to each particular tRNA. At this point, we did not distinguish between tRF-5s and tRF3 s. Low correlation between parental tRNA loci number and tRF-5 or tRF-3 abundance in A. thaliana, $O$. sativa or $P$. patens (Fig. 1a) was found. In several animal species, tRFs do not derive from abundant cellular tRNAs (Kumar et al. 2014). Similarly, we did not observed correlation between tRF abundance and tRNA gene copy number (Fig. 1a). For instance, A. thaliana tRNA-Arg ${ }^{\mathrm{CCG}}$ and $A$. thaliana
tRNA-Arg ${ }^{\mathrm{CCT}}$ have four and eight loci that mapped to 270 and 117 tRF reads, respectively. Conversely, tRNA-Pro ${ }^{\text {TGG }}$ and tRNA-Ser ${ }^{\mathrm{AGA}}$, which have 45 and 37 copies in the Arabidopsis genome, mapped only to 12 and 18 reads, respectively (data not shown). Our data is in agreement with those obtained in other species (Kumar et al. 2014) and suggests that plant tRF-5s and tRF-3s are not byproducts of random tRNA cleavage. In fact, each type of tRF seems to be generated by a specific parental tRNA and different kinds of enzymes could be acting on their cleavage (Hurto 2011).

To verify the distribution and frequency of 19 to $25 \mathrm{nt}$ tRF-5s and tRF-3s in the three species, we calculated the percentage of tRF isoforms from each size in each species and evaluated their expression (number of reads per million or RPM; Online Resource 3). While the distribution of tRF isoforms (19-25 nt in length) is similar among Arabidopsis, rice and moss, their expression is distinct in each species. For example, 19 to 20-nt tRFs are the most expressed isoforms in Arabidopsis, while in rice 25-nt tRFs seem to accumulate in higher levels (Online Resource 3). Next, we aligned tRFs from different sRNA libraries with parental tRNAs to verify whether tRFs have a bias regarding cleavage position. Similarly to other species (Lee et al. 2009; Li et al. 2012; Cai et al. 2013), we observed a peak of tRFs in $5^{\prime}$ termini of mature tRNAs in Arabidopsis, rice and moss, indicating that tRF asymmetric processing occurs in distinct plant species (Fig. 1b). Thus, tRF-5 seems to be the most expressed type of tRFs in plants (Fig. 1b). To estimate the conservation of tRF cleavage sites among plant precursor tRNAs, we compared 19 to $20 \mathrm{nt}$ tRF-5 and tRF-3 cleavage sites among Arabidopsis, rice and moss tRNAs. Similar to their precursors, plant tRF-5 and tRF-3 cleavage sites are generally conserved among evolutionary distant species (Online Resource 4). The precision with which individual tRFs are generated suggests that tRFs are not produced by random exonucleolytic digestion of tRNA precursors. In addition, because of the method of sRNA sequencing in these databases requires reverse transcriptase to read through the tRF into the adaptor sequence, tRNA modifications would, if anything, lower the number of reads observed, not create artificial short sequences.

To globally evaluate whether plant tRF expression varies among tissues/organs, we compared tRF-5 and tRF-3 RPM in Arabidopsis tissues/organs from publicly available databases (Online Resource 1). Both types of tRFs are present in all the tissues/organs analyzed, but independently of the tissues/organs evaluated, tRF-5s were the most abundant sRNAs observed in the libraries (Fig. 2a). This data agreed with recent work from Kumar et al. (2014), which found by meta-analysis that several tRFs differentially accumulate in distinct animal tissues/organs. To further investigate tRF expression in details, we selected two Arabidopsis tRF-5s (19-nt Arg ${ }^{\mathrm{CCT}}$ 5'-GCGCCUGUAGCUCAGUGGA 
(A)
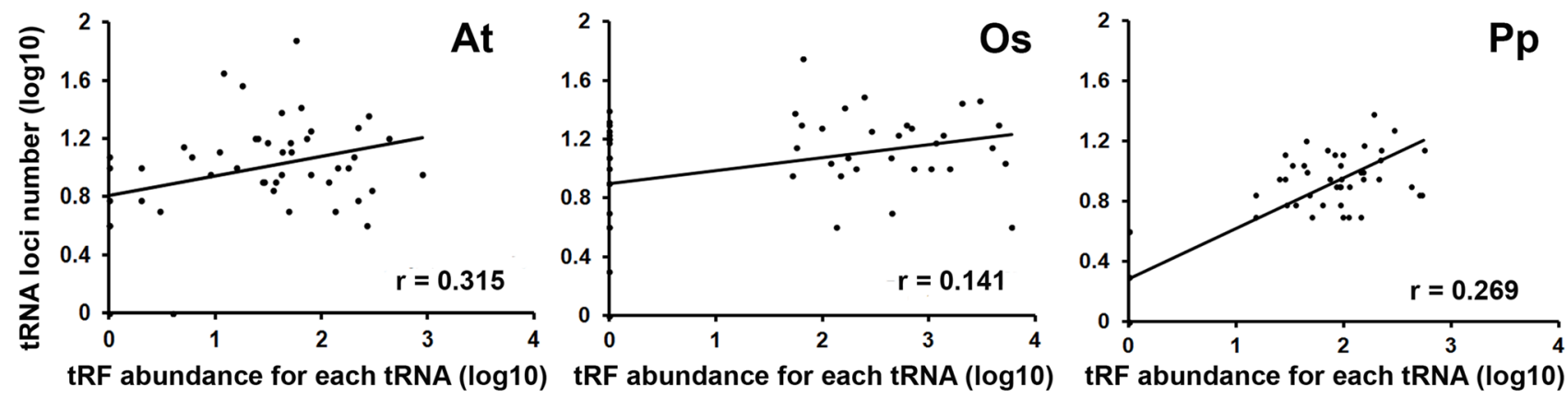

(B)
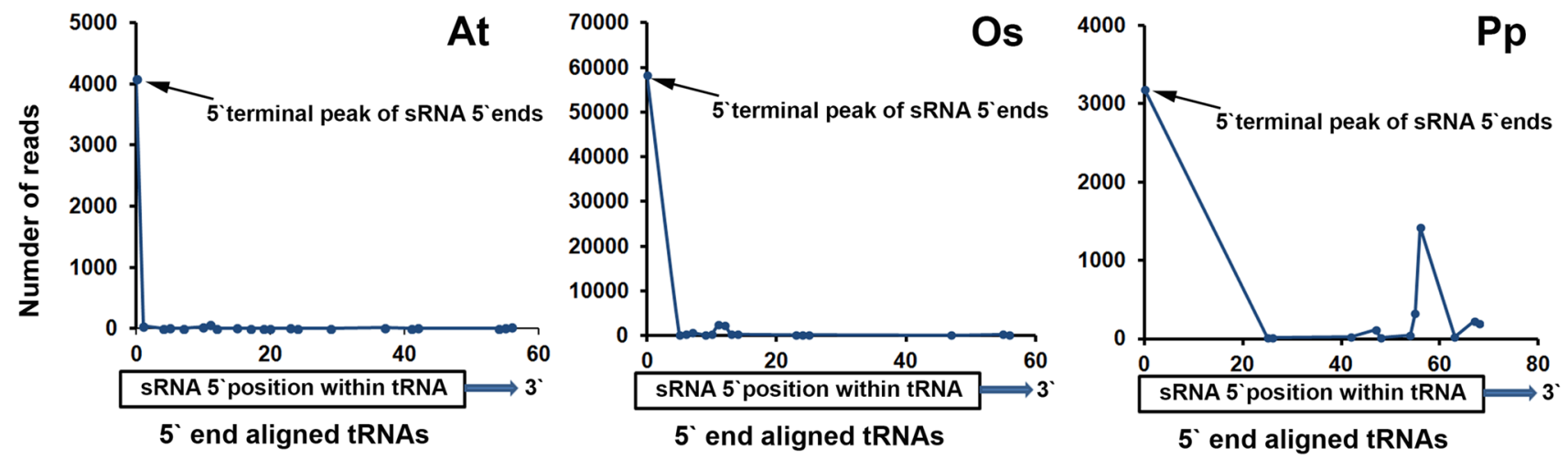

Fig. 1 Small RNAs (sRNAs) matching to the $5^{\prime}$ and $3^{\prime}$ termini of tRNAs (tRF-5s and tRF-3s) and their respective parental tRNAs are conserved across plant species. a Low or weak correlation between the abundance of conserved tRFs retrieved from sRNA libraries and the number of gene copy of their respective parental tRNAs in distinct plant species. The values were $\log _{10}$-transformed to facilitate comparisons. Pearson correlation coefficients (r) are shown for each species. b

and 20-nt $\operatorname{Arg}^{\mathrm{TCG}}$ 5'-GACCGCAUAGCGCAGTGGAU) and two tRF-3s (20-nt Ser ${ }^{\mathrm{GGA}}$ 5'-GAAUCCCUCUC UUUCCCCCA and 20-nt Tyr ${ }^{\text {GTA }} 5$ '-AAAUCCAGCUCGG CCCACCA) to perform in vivo studies by using stem-loop qRT-PCR approach (Fig. 2b). Stem-loop qRT-PCR is a standard technique to accurately quantify miRNAs (VarkonyiGasic et al. 2007; Ortiz-Morea et al. 2013), and it has been successfully used to quantify tRF expression in animals and plants (Chen et al. 2011; Li et al. 2016; Asha and Sonyia 2016; Olvedy et al. 2016). Moreover, it is highly unlikely that standard stem-loop qRT-PCR can amplify precursor or mature tRNAs, as post-transcriptional modifications are present in tRNAs, many of which play crucial roles in tRNA folding and function, inhibiting Watson-Crick base paring and thus arresting reverse-transcription (Kellner et al. 2010; Honda et al. 2015). All tRF transcripts were readily detectable by stem-loop qRT-PCR and further confirmed by agarose gel analysis and sequencing (Online Resource 5), indicating that they were authentic sRNAs (Fig. 2c). Interestingly, all four tRFs accumulated at higher levels in
The visualization of positions of all reads that map to tRNAs indicates that $5^{\prime}$ terminal of tRNAs produce the most number of fragments $\left(5^{\prime}\right.$ terminal peak). The tRNA position (with respect to its $5^{\prime}$ end) at which the $5^{\prime}$ end of the sRNA reads are matched are used, as depicted by the bottom illustration. At Arabidopsis thaliana, Os Oryza sativa, Pp Physcomitrella patens

leaf tissues comparing to flower tissues (Fig. 2c), reinforcing the idea that these small RNAs are not byproducts of random tRNA degradation in plants, similarly to what has been observed in animal cells (Kumar et al. 2014). Indeed, it has been reported lack of correlation between mature or precursor tRNA and their respective tRF abundance in different animal tissues (Soares et al. 2015), or under distinct conditions (Lee et al. 2009).

\section{Plant tRFs differentially accumulate under stress conditions}

Glucose is a key regulator for diverse processes in plants and has been shown to interfere with microRNA expression (Moore et al. 2003; Rook et al. 2006; Duarte et al. 2013). We therefore investigated whether glucose would modulate 19 to $25-\mathrm{nt}$ tRF expression by monitoring tRF-5 and tRF-3 RPM in untreated Arabidopsis seedlings and in seedlings treated with mannitol and glucose (Duarte et al. 2013). Only tRF-5s and tRF-3s present in 


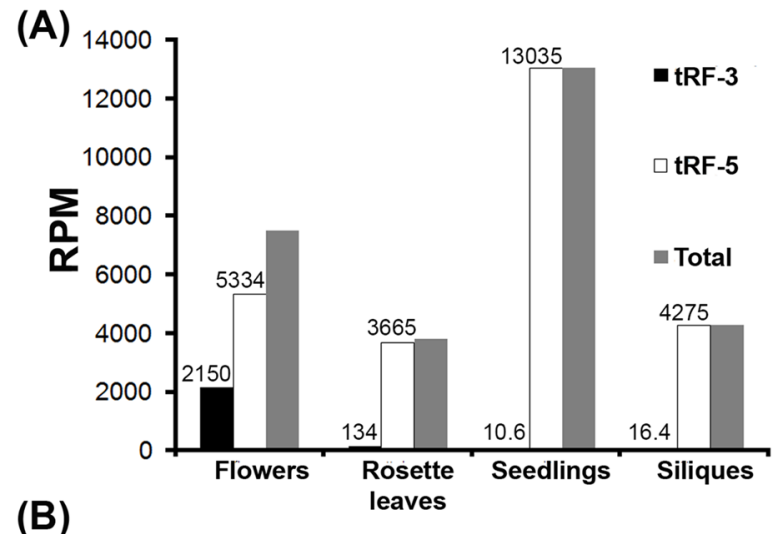

(B)
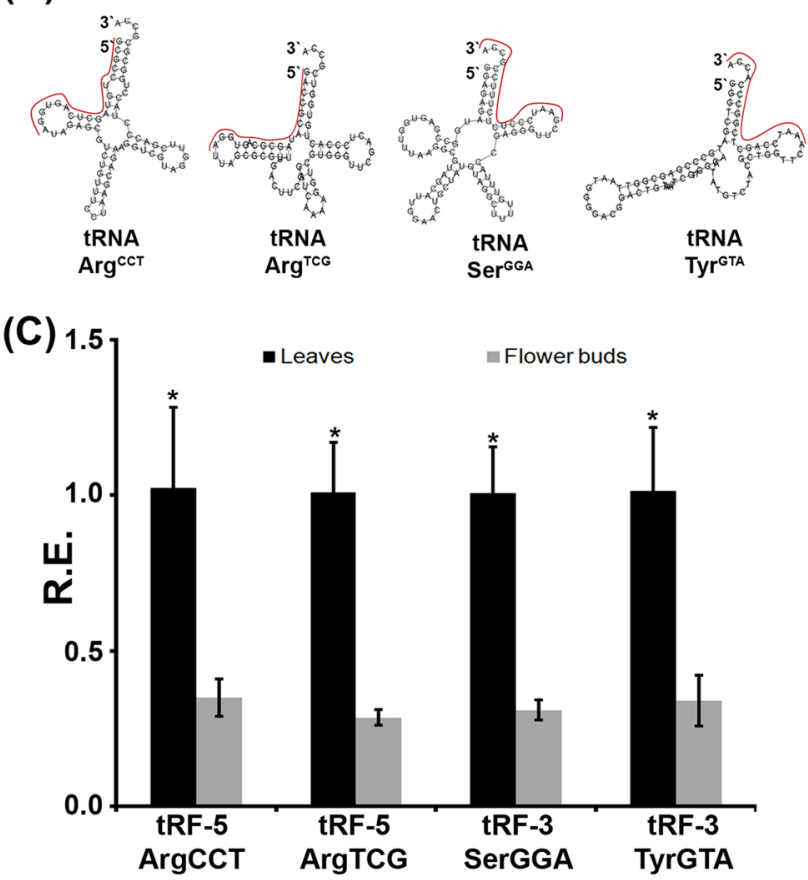

Fig. 2 Expression patterns of tRFs in tissues/organs of Arabidopsis. a Read counts for tRF-5s and tRF-3s in Arabidopsis tissues/organs from publicly available data (see Online Resource 1). The expression level of each type of tRF is shown on the Y-axis. b Representation of mature tRNA secondary structures analyzed in this study and their respective tRFs (red lines): 5'tRF-Arg ${ }^{\mathrm{CCT}}, 5^{\prime} \mathrm{tRF}$ Arg ${ }^{\mathrm{TCG}}, 3^{\prime} \mathrm{tRF}-\mathrm{Ser}^{\mathrm{GGA}}$, and 3'tRF $\mathrm{Tyr}^{\mathrm{GTA}}$. $\mathbf{c}$ Expression patterns of particular tRFs in 60-day-old Col-0 rosette leaves (leaves) and flower buds. Leaf expression values were set to one. Values are mean $\pm \mathrm{SD}$ of at least three biological replicates. Changes in transcript accumulation marked with an asterisk were considered significant with $p \leq 0.05$ according to Student's $t$ test (two tailed). Actin 2 was used as an endogenous control. RPM reads per million. $R E$ relative expression

both treatments were considered (Online Resource 6). Our data showed that the accumulation of most evaluated tRFs did not change (<twofold) in response to either glucose or mannitol treatments (Online Resource 6 ), suggesting that tRFs might not be directly involved in adjustments of growth and development triggered by sugar signaling. Conversely, the expression of several conserved tRFs seems to change ( $>$ twofold) in response to environmental stress conditions in Arabidopsis (LossMorais et al. 2013) and rice (Online Resource 7). For instance, 19-nt tRF-5 Arg ${ }^{\mathrm{CCT}}$ transcripts were detected at high levels (>twofold) in drought-treated Arabidopsis seedlings as well as in cold-treated rice inflorescences (Fig. 3a). These results suggested that the production and/ or accumulation of evolutionary conserved plant tRFs are regulated by abiotic stresses.

Oxidative stress can trigger the production of tRNA halves in several organisms, including plants (Thompson et al. 2008). Therefore, we analyzed whether tRFs also accumulate in Arabidopsis seedlings in response to this type of stress. We evaluated the expression of Arabidopsis tRF-5s $\mathrm{Arg}^{\mathrm{CCT}}$ (19 nt) and $\mathrm{Arg}^{\mathrm{TCG}}$ (20 nt) as well as tRF-3s Ser ${ }^{\mathrm{GGA}}$ $(20 \mathrm{nt})$ and $\mathrm{Tyr}^{\mathrm{GTA}}(20 \mathrm{nt})$ in seedlings which were grown under the presence or absence of hydrogen peroxide $\left(\mathrm{H}_{2} \mathrm{O}_{2}\right)$. Transcripts of tRF-5 Arg ${ }^{\text {TCG }}$ and tRF-3 Tyr ${ }^{\text {GTA }}$ accumulated at significant higher levels under oxidative stress conditions when compared with control (Fig. 3b), suggesting the accumulation of at least some tRFs in plant cells can be regulated by oxidative stress.

Stress conditions lead to changes in aminoacylation levels of tRNAs and uncharged tRNAs could participate in helping cells to survive under stressing environments. For instance, tRNAs could act as stress-associated signals, regulating cell death or being a substrate for tRF formation (Mei et al. 2010; Phizicky and Hopper 2010). Particular tRFs generated under stress conditions are capable of inhibiting protein synthesis (Gebetsberger et al. 2012). Although speculative, modulation of tRF expression by distinct abiotic stresses (Fig. 3; Online Resource 7) suggests similar roles of some tRFs during stress responses in plants.

\section{Biogenesis of most plant tRFs is not primarily dependent on DCLs}

Early studies suggested that the generation of some tRFs is dependent on DICER in certain human cell types (Cole et al. 2009; Haussecker et al. 2010). However, more recent works have confirmed that the canonical miRNA machinery is not crucial or it is even dispensable for the production of most tRFs in Phytophthora infestans, Drosophila, mice, and Schizosaccharomyces pombe (Asman et al. 2014; Buhler et al. 2008; Kumar et al. 2014). Therefore, we asked whether similar mi/siRNA-associated biogenesis pathways are involved in the generation of plant tRFs. We analyzed Arabidopsis libraries from mutants associated with small RNA machinery and RNA processing (Online Resource 1). We initially estimated tRF expression in flower tissues from $d c l 1-7$ and $d c l 2,3,4$ triple mutant and compared with WT from the same experiment, considering only those tRFs presented in all libraries (see "Materials and methods" for details). 
(A) At 19-nt tRF-5 ArgCCT

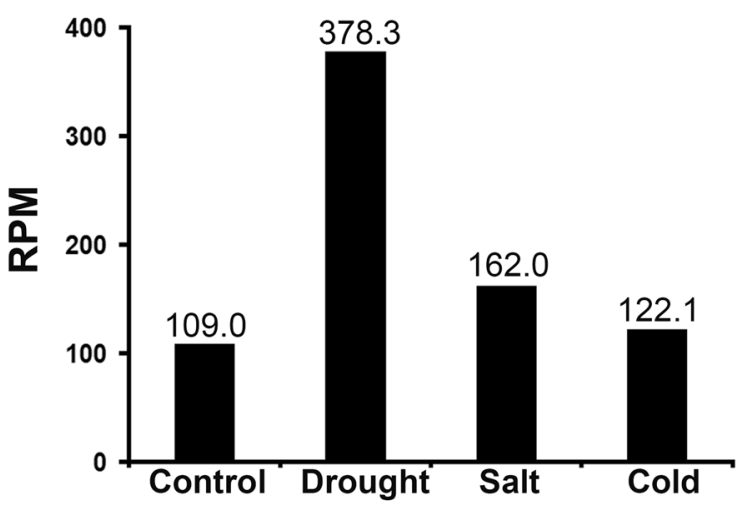

(B)

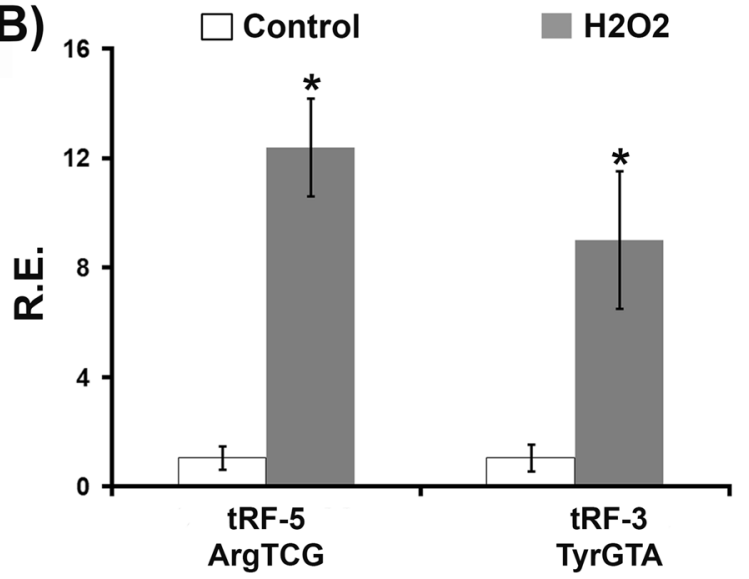

Os 19-nt tRF-5 ArgCCT
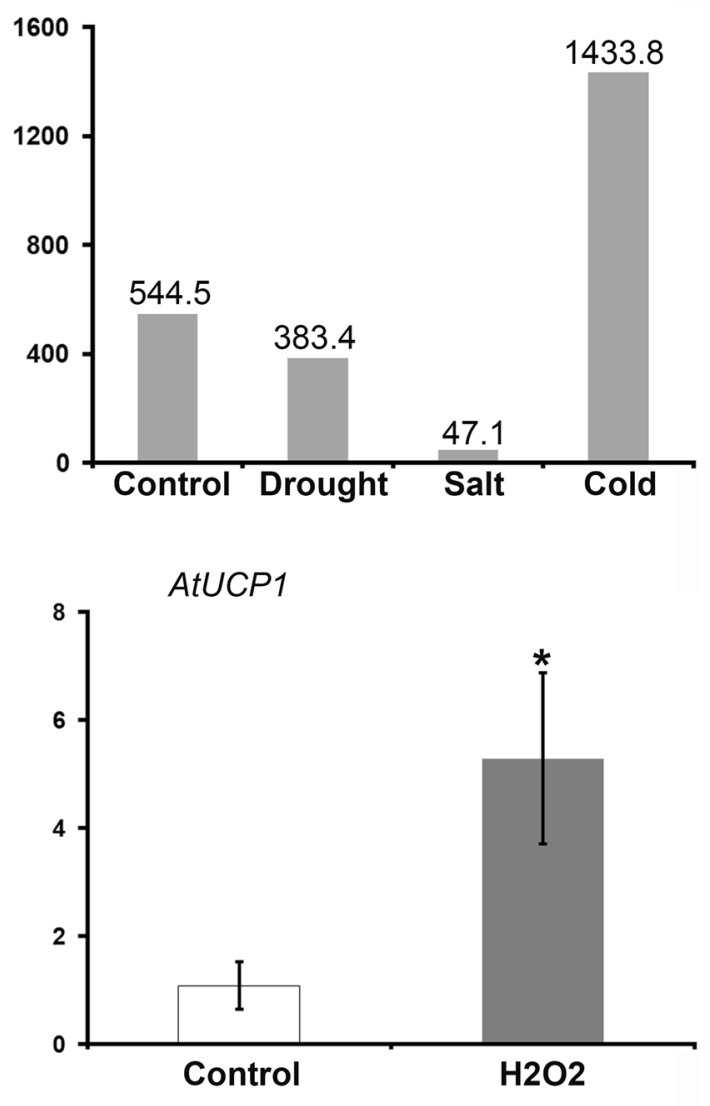

Fig. 3 Expression profiles of tRFs in response to abiotic stresses. a Read counts for $19 \mathrm{nt}$ tRF-5 Arg ${ }^{\mathrm{CCT}}$ in Arabidopsis (left) and rice (right) plants growing under diverse stress conditions (see Online Resource 1). The expression level of $19 \mathrm{nt} \mathrm{tRF}-5 \mathrm{Arg}^{\mathrm{CCT}}$ is shown on the $Y$-axis. b Expression profiles of tRF-5 Arg ${ }^{\text {TCG }}$ and tRF-3 Tyr $^{\text {GTA }}$ in response to oxidative stress. Expression values in the control (untreated plantlets) were set to one. Values are mean $\pm \mathrm{SD}$ of at least three biological

Interestingly, the majority ( $\sim 98 \%$ ) of tRF-5s and tRF-3s either did not change (<twofold) or was overrepresented in dcl1-7 libraries (Fig. 4a). Similar results were observed for most tRFs in the $d c l 2,3,4$ triple mutant libraries (Fig. $4 \mathrm{~b}$ ). Given that $d c l 1-7$ and $d c l 2,3,4$ mutants are defectives for DICER-like1 (DCL1) and DCL2,-3 and -4 function, respectively (Henderson et al. 2006), one would expected reduced cloning frequency of tRFs in $d c l$ mutants, as we observed for representative miRNAs in $d c l 1-7$ (Fig. 4c). Thus, our data suggest that these proteins are not primarily essential for the biogenesis of most Arabidopsis tRFs. In addition, the higher accumulation of several tRFs and miRNAs in $d c l 2,3,4$ mutant (Fig. 4) is most likely a result of the markedly reduction of tasiRNA and siRNA production in this triple mutant (Henderson et al. 2006), which would favor the sampling of other types of sRNAs. However, we cannot rule out the possibility that the biogenesis of particular tRFs may replicates. Changes in transcript accumulation marked with an asterisk were considered significant with $p \leq 0.05$ according to Student's $t$ test (two tailed). Actin2 was used as an endogenous control. RPM reads per million, $R E$ relative expression. Differential expression of Arabidopsis Uncoupling protein1 (AtUCP1) in response to oxidative stress (Begcy et al. 2011) is shown as a control for the $\mathrm{H}_{2} \mathrm{O}_{2}$ treatment

be DCL-dependent, as few Arabidopsis tRFs were underrepresented (>twofold) in both $d c l 1-7$ single and $d c l 2,3,4$ triple mutants (Fig. 4a, b).

To address individual processing of tRF-5s and tRF$3 \mathrm{~s}$, we looked into the accumulation of Arabidopsis tRF5s $\operatorname{Arg}^{\mathrm{CCT}}$ (19-nt) and $\mathrm{Arg}^{\mathrm{TCG}}$ (20-nt) and tRF-3s Ser ${ }^{\mathrm{GGA}}$ (20-nt) and Tyr ${ }^{\text {GTA }}(20-n t)$ in $d c l 1-7$ and $d c l 2,3,4$ as well as in hyll-2 and hyll-2,dcl1-21 double-mutant libraries, and compared them with their respective control experiments (Online Resource 1). HYPONATIC LEAVES1 (HYL1) is a double-strand RNA (dsRNA)-binding protein essential for miRNA biogenesis, assisting DCL1 in the pri-miRNA processing (Dong et al. 2008). Transcripts of 19-nt tRF-5 $\mathrm{Arg}^{\mathrm{CCT}}$ were detected only in $d c l 1-7$ and $d c l 2,3,4$ libraries, while 20-nt tRF-5 Arg ${ }^{\text {TCG }}$ and 20-nt tRF-3s Ser ${ }^{\mathrm{GGA}}$ and $\mathrm{Tyr}^{\mathrm{GTA}}$ were found in hyll-2 and hyll-2,dcll-21 libraries (Fig. 5a). Mutations of $d c l s$ or hyll did not decrease the 
(A)

\section{replicate \#1}

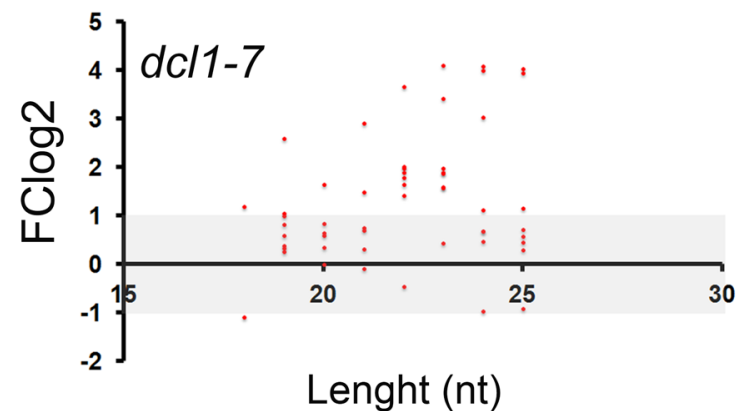

(B)

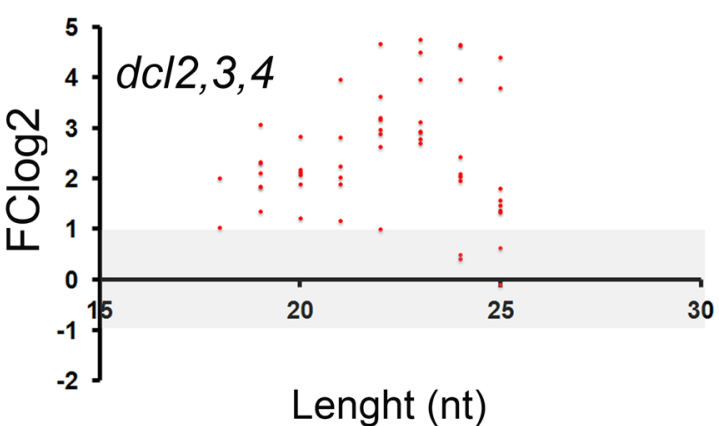

(C)

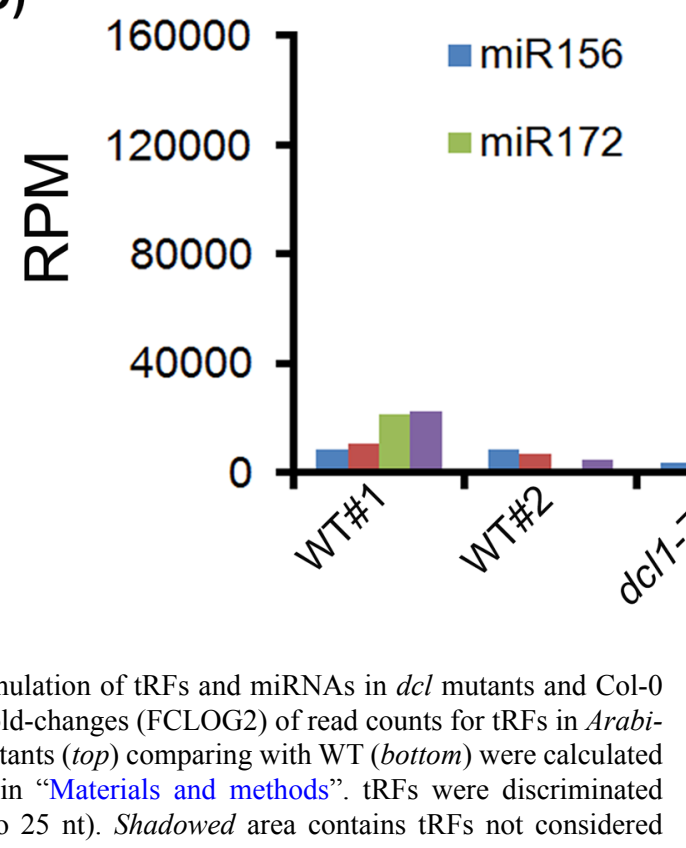

replicate \#2

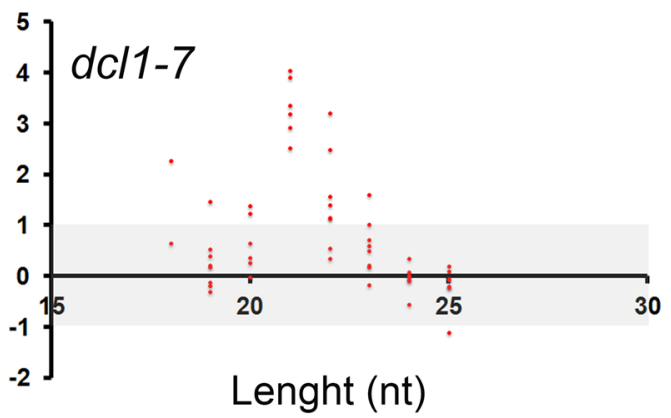

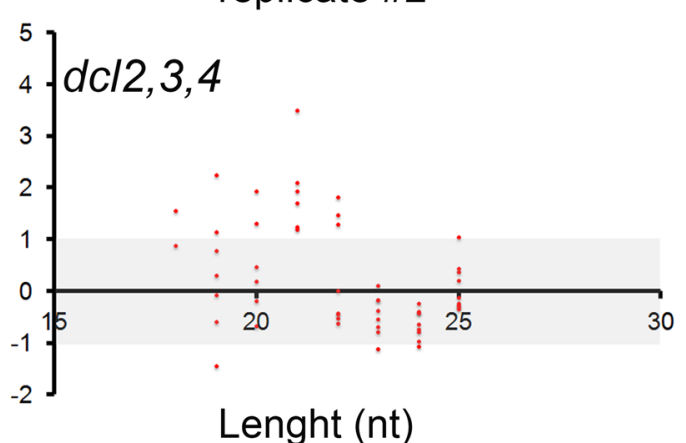

$\operatorname{miR} 166$

miR167

Fig. 4 Accumulation of tRFs and miRNAs in $d c l$ mutants and Col-0 (WT). a, b Fold-changes (FCLOG2) of read counts for tRFs in Arabidopsis dcl mutants (top) comparing with WT (bottom) were calculated as described in "Materials and methods". tRFs were discriminated by size (19 to $25 \mathrm{nt})$. Shadowed area contains tRFs not considered

expression of tRF-5s, and the frequency of tRF-3s Ser ${ }^{\mathrm{GGA}}$ and $\mathrm{Tyr}^{\mathrm{GTA}}$ was slightly reduced in hyll and hyll-2,dcll-21 libraries (Fig. 5a). To confirm the in silico analyses, we differentially expressed (<twofold). c Read counts for microRNAs (miR156, miR172, miR166, and miR167) in Arabidopsis dcl mutants and WT. All read counts were retrieved from publicly available data (see Online Resource 1). RPM reads per million

evaluated transcript accumulation of 19-nt tRF-5 Arg ${ }^{\mathrm{CCT}}$, 20-nt tRF-5 Arg ${ }^{\text {TCG }}$ and 20-nt tRF-3s Ser ${ }^{\text {GGA }}$ and Tyr ${ }^{\text {GTA }}$ in seedlings of Col-0 (WT), dcl, and hyll mutants. In general, 

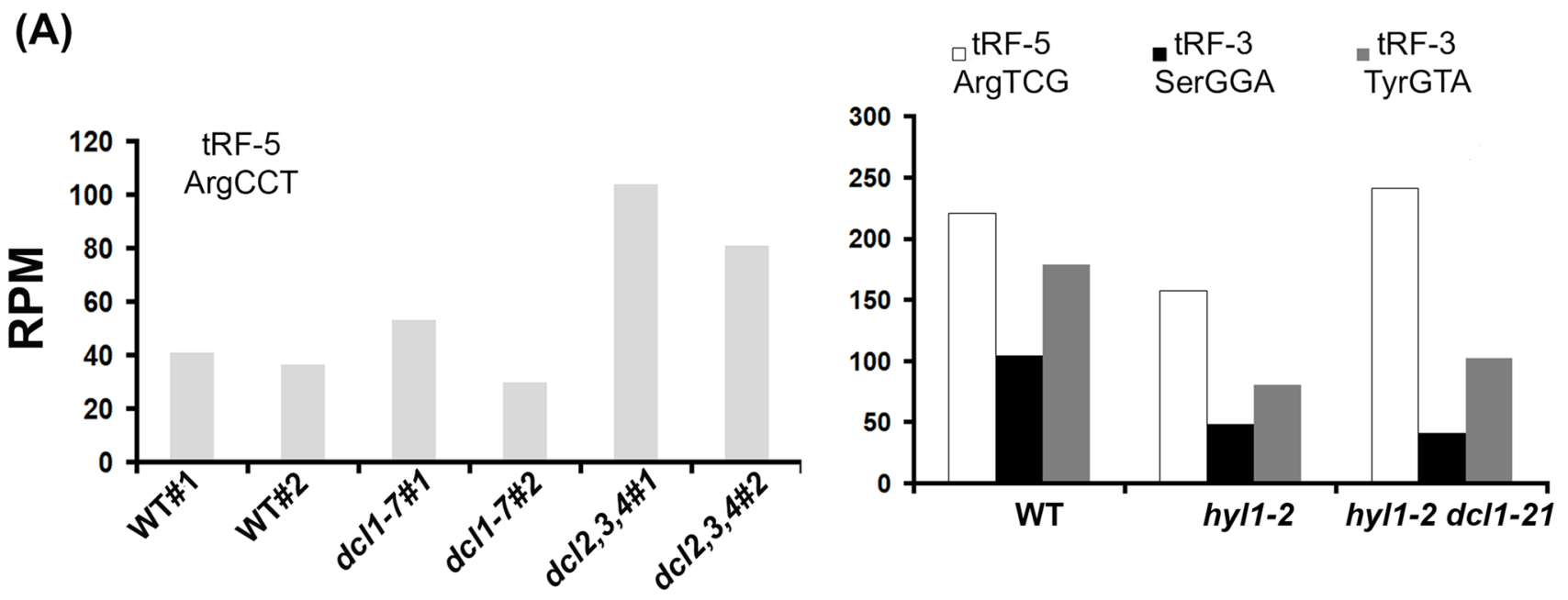

(B)

(C)
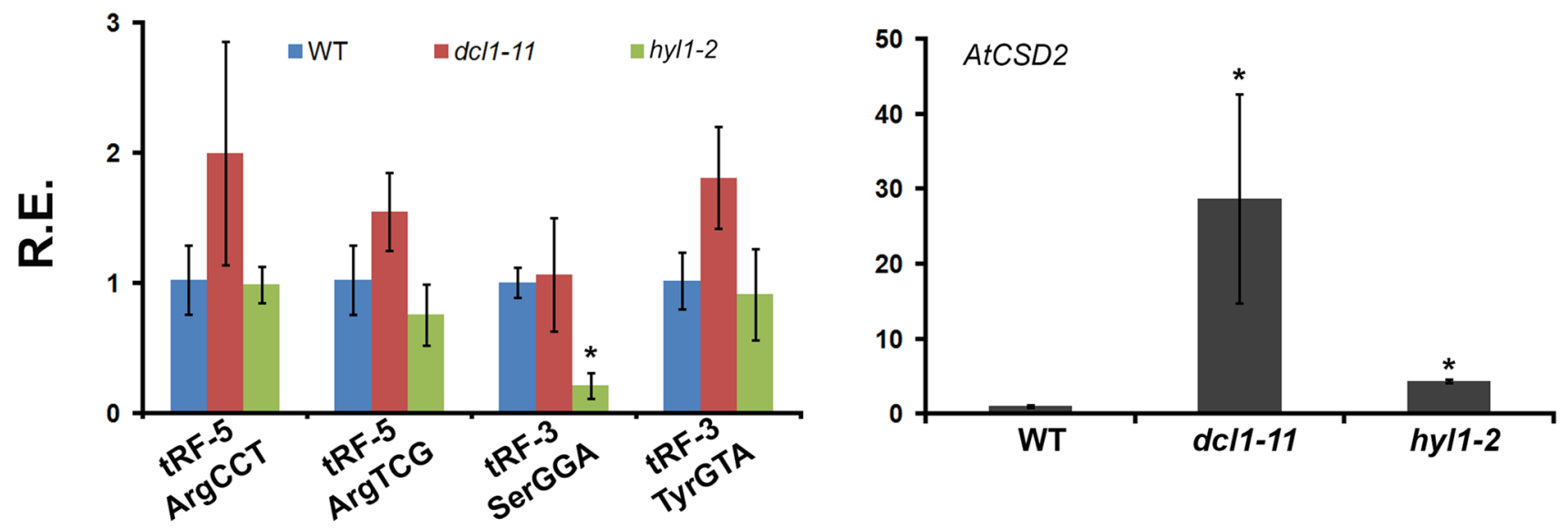

Fig. 5 Accumulation of specific tRFs in seedlings of Col-0 (WT) as well as $d c l$ and hyll mutants. a Read counts for tRF-5s and tRF-3s in Arabidopsis dcll, dcl2,3,4, hyll, and hyll,dcll mutants comparing with WT. All read counts were retrieved from publicly available data (see Online Resource 1). b Expression patterns of tRF-5s Arg ${ }^{\mathrm{CCT}}$ and $\mathrm{Arg}^{\mathrm{TCG}}$ as well as tRF-3s Ser ${ }^{\mathrm{GGA}}$ and Tyr ${ }^{\mathrm{GTA}}$ in seedlings of Col-0 (WT) and dcll-11 and hyll-2 mutants. Expression values in WT were set to one. Values are mean \pm SD of at least three biological replicates.

the expression of these tRFs did not change significantly in the mutants when comparing with WT, though some tRFs seemed to overaccumulate in $d c l$ mutants (Fig. 5b; Online Resource 8). In the context of all mutants, we propose that DCLs are not primarily involved in the generation of most tRFs in Arabidopsis. However, it is possible that particular components of the miRNA biogenesis machinery (i.e., HYL1) participate in the generation of tRF-3s, as hyll-2 mutation decreased significantly tRF-3 Ser ${ }^{\mathrm{GGA}}$ transcript levels (Fig. 5). tRF biogenesis in other plant species seems not to be dependent on DCLs as well, as the expression of conserved tRFs in rice did not change (<twofold) in $d c l$ mutants when comparing with their respective control experiments (Online Resource 9).
Changes in transcript accumulation marked with an asterisk were considered significant with $p \leq 0.05$ according to Student's $t$ test (two tailed). Actin2 was used as an endogenous control. RPM reads per million. $R E$ relative expression. $\mathbf{c}$ Differential accumulation of miR398targeted Arabidopsis COPPER/ZINC SUPEROXIDE DISMUTASE2 (AtCSD2) (Smeets et al. 2009) is shown as a control for dcll-11 and hyll-2 mutants

This study raises at least two important questions about tRF biogenesis in general: If DCLs in plants or DICER and DROSHA in animals (Kumar et al. 2014) are not crucial for tRF generation then which proteins are? Do all organisms generate tRFs by the same pathway? There are still controversies regarding what enzymes are important for tRF biogenesis in different organisms. For example, RNase $\mathrm{Z}$ is responsible for 3'-pre-tRNA cleavage, while tRNA-halves could be processed by Angiogenin in mammals, Rny1 in yeast and PrrC, colicin D and colicin E5 in bacteria (Hackenberg et al. 2012). At least for some human tRF-5s, DICER was shown to be responsible for their processing, but only in $\mathrm{HeLa}$ cells. More puzzling is the fact that DICER is also able to process particular tRF-3s in HeLa cells, as do Angiogenin 
and other RNases A in a DICER-independent pathway (For review, see Raina and Ibba 2014).

Our data suggested that the generation of most Arabidopsis tRF-5s and tRF-3s is only partially dependent on DCLs (Figs. 4, 5). Therefore, we decided to investigate whether other plant ribonucleases could be candidates to participate in tRF biogenesis or accumulation. Angiogenin has been shown to participate in the processing of tRNAs to generate tRFs in human cells (Yamasaki et al. 2009; Ivanov et al. 2011). Plants do not have Angiogenin-like ribonucleases, but some members of the ancient superfamily of ribonucleases T2/S might have similar functions. One such member may be the enzyme S-like Ribonuclease 1 (RNS1) (Bariola et al. 1994; MacIntosh et al. 2010). RNS1 is associated with stress responses, being upregulated in several cell types under these conditions. Nevertheless, RNS1 is also expressed under non-stress conditions in flowers and at lower levels in leaves and roots of young seedlings (Hillwig et al. 2008).

RNS1 is conserved across evolutionary distant plant species (Online Resource 10), being a good ribonuclease candidate to be involved in tRF biogenesis. Thus, we asked whether RNS1 could participate in Arabidopsis tRF biogenesis. To do so, we initially identified a T-DNA insertion line from TAIR collection (https://www.Arabidopsis.org/), hereafter referred to as rsn1-1 (salk_087165C). Interestingly, the T-DNA insertion in the promoter region of RNS1 locus (Online Resource 11) promoted high RNS1 transcript accumulation in leaf tissues of homozygous $r s n 1$ 1 plants (Fig. 6a). RNS1 transcript accumulation is tightly regulated at transcriptional levels in response to $\mathrm{ABA}$ and wounding stress in Arabidopsis (Hillwig et al. 2008). The T-DNA insertion in $r s n 1-1$ plants is located within the proximal $1000 \mathrm{nt}$ of the promoter sequence, in which several

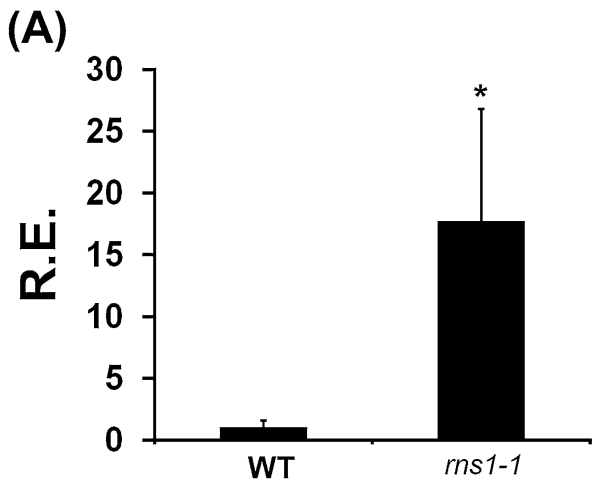

Fig. 6 RNS1 might be involved in the biogenesis of tRFs in Arabidopsis. a Expression pattern of RNS1 in Col-0 (WT) and rns 1-1 mutant plants. b Expression patterns of tRF-5s Arg ${ }^{\mathrm{CCT}}$ and $\mathrm{Arg}^{\mathrm{TCG}}$ as well as tRF-3s Ser ${ }^{\text {GGA }}$ and Tyr ${ }^{\text {GTA }}$ in Col-0 (WT) and rns 1-1 mutant plants. Expression values in WT were set to one. Values are mean \pm SD of wound-responsive and ABA-responsive elements were found (Hillwig et al. 2008). It is possible that RNS1 transcripts are more abundant in $r s n 1-1$ leaf tissues due to the disruption of some of these elements, which likely allows high transcript accumulation under non-stress conditions (Fig. 6a).

Given that tRF-5s $\operatorname{Arg}^{\mathrm{CCT}}$ (19-nt) and $\operatorname{Arg}^{\mathrm{TCG}}$ (20-nt) as well as tRF-3s Ser ${ }^{\text {GGA }}$ (20-nt) and $\mathrm{Tyr}^{\mathrm{GTA}}$ (20-nt) accumulated at high levels in rosette leaves from 60-day-old Col-0 plants (Fig. 2c), this growth stage was chosen to compare their accumulation patterns in rsn1-1 and WT (Col-0) plants. Transcripts of both tRF-5s and tRF-3s showed higher accumulation levels in mutant leaves when comparing with WT (Fig. 6b). Interestingly, Arabidopsis RNS1 has been recently shown to be able to generate both tRNA halves and tRFs (Megel et al. 2015). Our data and from others (Megel et al. 2015) suggest the possibility that RNS1 is involved, perhaps indirectly, in the processing and degradation of tRFs. However, future studies are needed to evaluate at whole-genome level the possible impact, if any, of RSN1on tRF biogenesis in plants.

\section{Plant AGOs incorporate tRFs but have few potential targets}

To evaluate if plant 19 to $25-\mathrm{nt} \mathrm{tRF}-5 \mathrm{~s}$ and tRF-3s associate with Argonaute proteins (AGOs), we inspected tRF accumulation (given in reads per million or RPM) in publicly available Arabidopsis AGO immunoprecipitation (AGO-IP) libraries (Online Resource 1). To focus on the most reproducible set of tRFs, we considered associated with AGOs those tRFs with at least 10 RPM (see "Materials and methods"). We identified 19 to 25 -nt tRF-5s and tRF-3s that were associated with distinct plant AGOs (Fig. 7). However, tRF

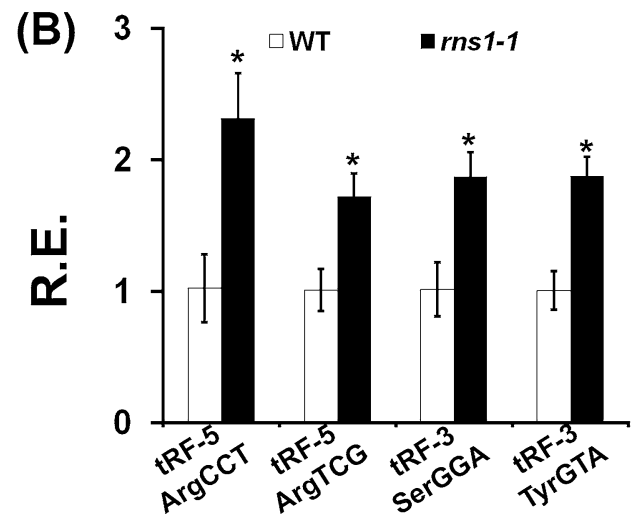

at least three biological replicates. Changes in transcript accumulation marked with an asterisk were considered significant with $p \leq 0.05$ according to Student's $t$ test (two tailed). Actin2 was used as an endogenous control. $R E$ relative expression 
read counts were lower when comparing to incorporated miRNAs, mainly for AGO1 (Online Resource 12). We found that 19-nt tRF-5s were the most abundant type of tRF incorporated into Arabidopsis AGOs, in agreement with previous results (Loss-Morais et al. 2013). Nevertheless, we detected bias towards particular tRF sizes in distinct AGOs (Fig. 7a). For example, AGO1 incorporated high levels of 19 and 20-nt tRF-5s, while 19-nt tRF-5s were preferentially found to be associated with AGO2. For AGO4, the most abundant types of tRF-5s were 19, 24, and 25 nucleotides in length. Finally, AGO5 incorporates 19-nt tRF-5s at higher levels (Fig. 7a). Similarly to Arabidopsis, 19-nt tRF-5s were highly abundant in rice AGO-IP libraries. Interestingly, rice AGOs seem to be associated with specific types of 19-nt tRF-5s, of which the conserved tRF-5s Arg ${ }^{\mathrm{CCT}}$ and $\mathrm{Arg}^{\mathrm{TCG}}$ were the most abundant (Fig. 7b; Loss-Morais et al. 2013). Our data showed that conserved tRFs are incorporated into AGOs across plant species, indicating that they may have biological roles in sRNA-mediated silencing processes.

To investigate the possible role(s) of plant tRFs in miRNA-mediated silencing pathways, we predicted target candidates for the most abundant Arabidopsis AGO1associated tRFs by using psRNATarget (Dai and Zhao 2011) based on sequence complementarity coupled with
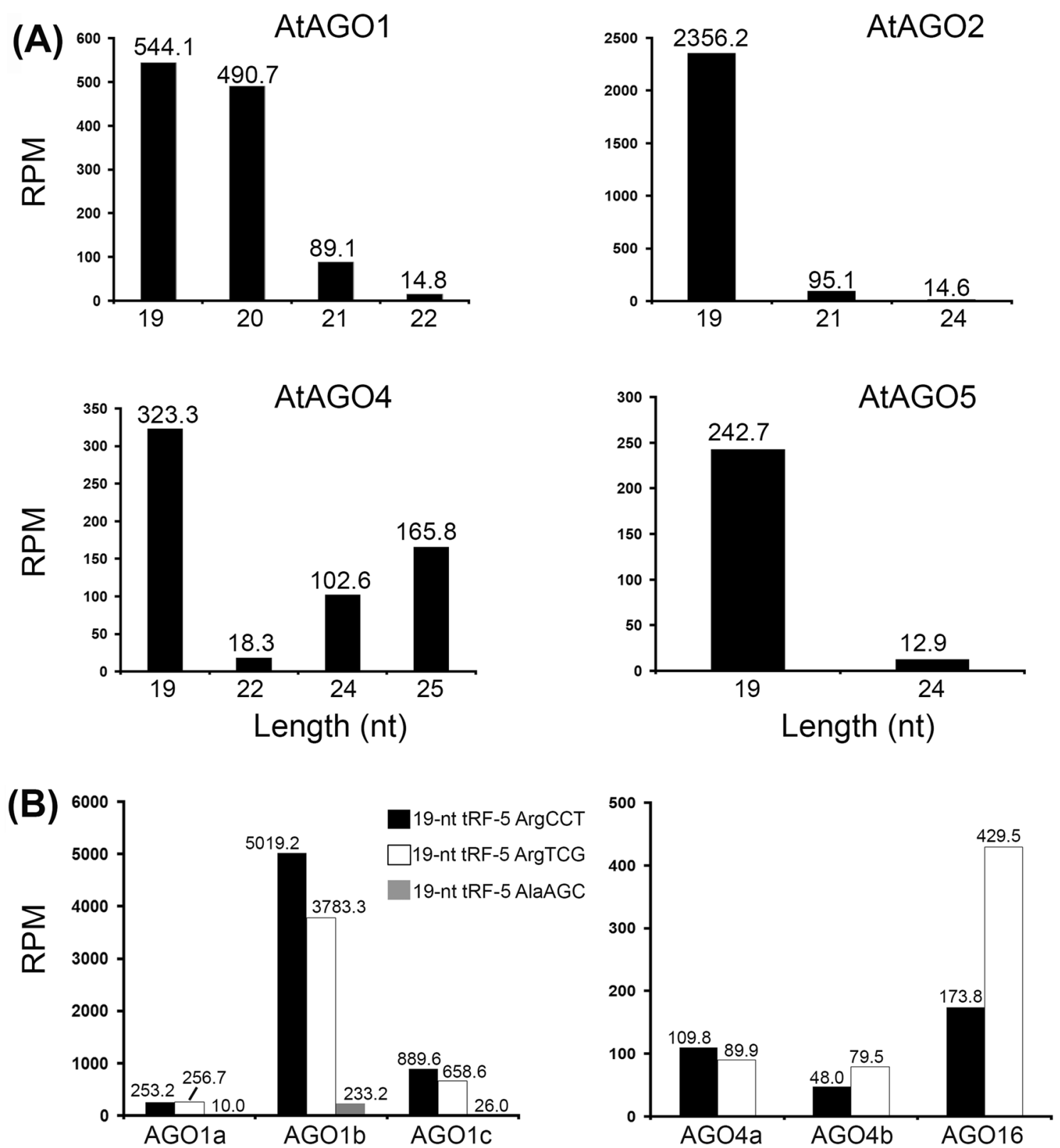

Fig. 7 tRF-5s and tRF-3s associated with plant AGOs. Read counts for tRF-5s and tRF-3s in Arabidopsis (a) and rice (b) AGO-IP data (see Online Resource 1). tRFs were discriminated by size (19-25 nt). RPM reads per million 
degradome analyses. We employed more stringent cutoff threshold for the Maximum expectation (E) parameter (range 0-2.0; Dai and Zhao 2011) to minimize false positive target prediction. Few potential targets were found for some tRF-5s, of which most presented a low number of PARE (Parallel analysis of RNA end) signatures when comparing with miRNA target-associated PARE signatures (Online Resource 13). This suggests that tRFs may act through regulatory mechanisms other than mRNA cleavage or degradation.

Plant tRFs might play a role in DNA and chromatin modifications as we identified 24-nt tRFs associated with AGO4 (Fig. 7). AGO4 is localized in the nucleus and associates predominantly with $24 \mathrm{nt}$ small RNAs having $5^{\prime}$ terminal $\mathrm{A}$ in order to mediate DNA methylation in transposons and a few coding loci (Mi et al. 2008; Li et al. 2008). tRF-3 Leu ${ }^{\text {CAA }}$ (5'-AAGAGGGCG TGGGTTCAAACCCCA) is $24 \mathrm{nt}$ in length and it is associated with AGO4 ( 28 RPM; data not shown). Through BLASTn analysis, we looked for tRF-3 Leu ${ }^{\mathrm{CAA}}$ potential target regions in the Arabidopsis genome. Interestingly, tRF-3 Leu ${ }^{\mathrm{CAA}}$ was extensively complementary to upstream region of SUPPRESSOR OF FRIGIDA4 (SUF4) locus (Online Resource 13), which acts as a transcriptional activator of FLOWERING LOCUS C (FLC) to regulate flowering (Kim et al. 2006). Moreover, SUF4 also interacts directly with MED18 (Mediator 18), a transcriptional cofactor complex that affects the levels of H3K36me3, a histone mark for active regions (Lai et al. 2014). Perhaps tRF-3 Leu ${ }^{\mathrm{CAA}} / \mathrm{AGO} 4-$ mediated methylation of SUF4 might be important to indirectly promote flowering. Some human tRF-3s were shown to associate with AGOs, potentially acting as miRNAs as well as siRNAs (Haussecker et al. 2010). Although speculative, we propose that particular 24-nt tRF-3 small RNAs may function as "guides" to drive AGO4 to specific loci to recruit epigenetic machinery for de novo DNA methylation (Rose and Klose 2014).

A few additional biological functions have been linked to tRFs thus far. For instance, HIV (human immunodeficiency virus) uses the $3^{\prime}$ end of the human tRNA Lys as a primer for its retrotranscriptase and DNA synthesis (Yeung et al. 2009). Other report showed that tRF-5s can inhibit protein synthesis in archeon Haloferax volcanii (Gebetsberger et al. 2012). Additionally, some 19-nt tRF5 s from human cells can inhibit protein synthesis in vivo and in vitro without a complementary sequence on the mRNA, but a GG motive at $3^{\prime}$ end is required (Gebetsberger et al. 2013). These data indicate the possibility that tRFs, besides canonical RNAi mechanisms, likely have non-canonical mechanisms to control gene expression or modulate protein biosynthesis (Sobala and Hutvagner 2013).

\section{Conclusions}

Recently, novel studies on animals have shown how versatile tRNAs can be in terms of biological functions. This may be in part due to the fact that tRNAs are precursors of small RNAs such as tRFs. Our study shed some light on how diverse tRFs are in plants and showed that plant and animal tRFs shared common features. For example, plant tRFs are conserved across evolutionary distant species and are generated by asymmetric processing. Moreover, biogenesis of most plant tRFs seemed to occur through non-canonical mi/ siRNA pathways, similarly to tRF biogenesis in several animal species (Kumar et al. 2014). Additionally, like animal tRFs (Kumar et al. 2014), plant tRF-5s and tRF-3s are also incorporated into AGOs but seem to have much less targets.

Because of their association with different AGOs, particularly AGO4, it is conceivable that at least some plant tRFs act similarly as sidRNAs, a recently described new class of siRNAs independent of DCLs, which are incorporated into AGO4 to trigger de novo DNA methylation in Arabidopsis (Ye et al. 2015). Moreover, interplay between tRFs and other sRNAs might be important to fine tune gene expression and protein biosynthesis in plant cells. More specifically, 19-25 nt tRFs may be incorporated into distinct plant AGOs in particular tissues, developmental stages and/ or physiological conditions depending upon tRF abundance as well as $A G O$ expression patterns and AGO sorting. In animal cells, modulation of tRF levels by AGOs had minor effects on the abundance of miRNAs, but more pronounced changes in the silencing activities of both miRNAs and siRNAs, indicating that tRFs may play a role in the global control of sRNA silencing (Haussecker et al. 2010). Whether plant and animal tRFs share such global sRNA silencingmediated control of gene expression remains a major topic for future studies.

Acknowledgments We thank Dr. Nogueira's lab members for helpful discussions. This work was supported by the State of Sao Paulo Research Foundation, FAPESP, and National Council of Technological and Scientific Development $(\mathrm{CNPq})$, Brazil (Grants No. 480628/2012-2 and 07/58289-5). CSA was a recipient of a fellowship (No. 2011/19512-6) from the State of Sao Paulo Research Foundation, FAPESP, Brazil.

Author contributions CSA, GTD, and VFP carried out the molecular biology studies and analyzed the data; RV and CSA carried out the bioinformatic analyzes and MV helped to analyze data; FTSN designed and coordinated the study, and FTSN and CSA wrote the manuscript. All authors read and approved the final manuscript.

\section{References}

Asha S, Soniya EV (2016) Transfer RNA derived small RNAs targeting defense responsive genes are induced during Phytophthora 
capsici infection in Black Pepper (Piper nigrum L.). Front Plant Sci 7:1-16. doi:10.3389/fpls.2016.00767

Asman A, Vetukuri RR, Jahan SN, Fogelqvist J, Corcoran P, Avrova AO, Whisson SC, Dixelius C (2014) Fragmentation of tRNA in Phytophthora infestans asexual life cycle stages and during host plant infection. BMC Microbiol 14:308. doi:10.1186/ s12866-014-0308-1

Bariola PA, Howard CJ, Taylor CB, Verburg MT, Jaglan VD, Green PJ (1994) The Arabidopsis ribonuclease gene RNS1 is tightly controlled in response to phosphate limitation. Plant J 6:673-685. doi:10.1046/j.1365-313X.1994.6050673.x

Barrett T, Wilhite SE, Ledoux P, Evangelista C, Kim IF, Tomashevsky M, Marshall KA, Phillippy KH, Sherman PM, Holko M, Yefanov A, Lee H, Zhang N, Robertson CL, Serova N, Davis S, Soboleva A (2013) NCBI GEO: archive for functional genomics data sets-Update. Nucleic Acids Res 41:991-995. doi:10.1093/nar/ gks1193

Begcy K, Mariano ED, Mattiello L, Nunes AV, Mazzafera P, Maia IG, Menossi M (2011) An Arabidopsis mitochondrial uncoupling protein confers tolerance to drought and salt stress in transgenic tobacco plants. PLoS One 6:e23776. doi:10.1371/journal. pone. 0023776

Buhler M, Spies N, Bartel DP, Moazed D (2008) TRAMP-mediated RNA surveillance prevents spurious entry of RNAs into the Schizosaccharomyces pombe siRNA pathway. Nat Struct Mol Biol 15:1015-1023. doi:10.1038/nsmb.1481

Cai P, Piao X, Hao L, Liu S, Hou N, Wang H, Chen Q (2013) A deep analysis of the small non-coding RNA population in Schistosoma japonicum eggs. PLoS One. doi:10.1371/journal.pone.0064003

Chan PP, Lowe TM (2009) GtRNAdb: a database of transfer RNA genes detected in genomic sequence. Nucleic Acids Res 37:9397. doi:10.1093/nar/gkn787

Chen C-J, Liu Q, Zhang Y-C, Qu L-H, Chen Y-Q, Gautheret D (2011) Genome-wide discovery and analysis of microRNAs and other small RNAs from rice embryogenic callus. RNA Biol 8:538-547. doi:10.4161/rna.8.3.15199

Cole C, Sobala A, Lu C, Thatcher SR, Bowman A, Brown JWS, Green PJ, Barton GJ, Hutvagner G (2009) Filtering of deep sequencing data reveals the existence of abundant Dicer-dependent small RNAs derived from tRNAs. RNA 15:2147-2160. doi:10.1261/ rna.1738409

Dai X, Zhao PX (2011) PsRNATarget: a plant small RNA target analysis server. Nucleic Acids Res 39:155-159. doi:10.1093/nar/ gkr319

Dong Z, Han M-H, Fedoroff N (2008) The RNA-binding proteins HYL1 and SE promote accurate in vitro processing of pri-miRNA by DCL1. Proc Natl Acad Sci USA 105:9970-9975. doi:10.1073/ pnas.0803356105

Duarte GT, Matiolli CC, Pant BD, Schlereth A, Scheible WR, Stitt M, Vicentini R, Vincentz M (2013) Involvement of microRNArelated regulatory pathways in the glucose-mediated control of Arabidopsis early seedling development. J Exp Bot 64:43014312. doi:10.1093/jxb/ert239

Emara MM, Ivanov P, Hickman T, Dawra N, Tisdale S, Kedersha N, Hu GF, Anderson P (2010) Angiogenin-induced tRNA-derived stress-induced RNAs promote stress-induced stress granule assembly. J Biol Chem 285:10959-10968. doi:10.1074/jbc. M109.077560

Farazi T a, Juranek S a, Tuschl T (2008) The growing catalog of small RNAs and their association with distinct Argonaute/Piwi family members. Development 135:1201-1214. doi:10.1242/ dev.005629

Fu Y, Lee I, Lee YS, Bao X (2015) Small non-coding transfer RNADerived RNA fragments (tRFs): their biogenesis, function and implication in human diseases. Genomics Inform 13:94. doi:10.5808/GI.2015.13.4.94
Gebetsberger J, Polacek N (2013) Slicing tRNAs to boost functional ncRNA diversity. RNA Biol 10:1798-1806. doi:10.4161/ rna. 27177

Gebetsberger J, Zywicki M, Künzi A, Polacek N (2012) tRNAderived fragments target the ribosome and function as regulatory non-coding RNA in Haloferax volcanii. Archaea 2012:1-11. doi:10.1155/2012/260909

German MA, Pillay M, Jeong D-H, Hetawal A, Luo S, Janardhanan P, Kannan V, Rymarquis LA, Nobuta K, German R, De Paoli E, Lu C, Schroth G, Meyers BC, Green PJ (2008) Global identification of microRNA-target RNA pairs by parallel analysis of RNA ends. Nat Biotechnol 26:941-946. doi:10.1038/nbt1417

Hackenberg M, Huang P-J, Huang C-Y, Shi B-J, Gustafson P, Langridge P (2012) A comprehensive expression profile of MicroRNAs and other classes of non-coding small RNAs in barley under phosphorous-deficient and -sufficient conditions. DNA Res 1-17. doi:10.1093/dnares/dss037

Haussecker D, Huang Y, Lau A, Parameswaran P, Fire AZ, Kay MA (2010) Human tRNA-derived small RNAs in the global regulation of RNA silencing. RNA 16:673-695. doi:10.1261/rna.2000810

Henderson IR, Zhang X, Lu C, Johnson L, Meyers BC, Green PJ, Jacobsen SE (2006) Dissecting Arabidopsis thaliana DICER function in small RNA processing, gene silencing and DNA methylation patterning. Nat Genet 38:721-725. doi:10.1038/ng1804

Hillwig MS, LeBrasseur ND, Green PJ, MacIntosh GC (2008) Impact of transcriptional, ABA-dependent, and ABA-independent pathways on wounding regulation of RNS1 expression. Mol Genet Genomics 280:249-261. doi:10.1007/s00438-008-0360-3

Honda S, Shigematsu M, Morichika K, Telonis AG, Kirino Y (2015) Four-leaf clover qRT-PCR: a convenient method for selective quantification of mature tRNA. RNA Biol 12:501-508. doi:10.10 80/15476286.2015.1031951

Hopper AK, Shaheen HH (2008) A decade of surprises for tRNA nuclear-cytoplasmic dynamics. Trends Cell Biol 18:98-104. doi:10.1016/j.tcb.2008.01.001

Hsieh L-C, Lin S-I, Shih AC-C, Chen J-W, Lin W-Y, Tseng C-Y, Li W-H, Chiou T-J (2009) Uncovering small RNA-mediated responses to phosphate deficiency in Arabidopsis by deep sequencing. Plant Physiol 151:2120-2132. doi:10.1104/pp.109.147280

Hurto RL (2011) Unexpected functions of tRNA and tRNA processing enzymes. In: Collins LJ (ed) RNA infrastructure and networks. Landes Bioscience and Springer Science, pp 137-155

Ivanov P, Emara MM, Villen J, Gygi SP, Anderson P (2011) Angiogenin-induced tRNA fragments inhibit translation initiation. Mol Cell 43:613-623. doi:10.1016/j.molcel.2011.06.022

Kellner S, Burhenne J, Helm M (2010) Detection of RNA modifications. RNA Biol 7(2):237-247

Kenrick P, Crane PR (1997) The origin and early evolution of plants on land. Nature 389:33-39. doi:10.1038/37918

Kim S, Choi K, Park C, Hwang HJ, Lee I (2006) SUPPRESSOR OF FRIGIDA4, encoding a C2H2-Type zinc finger protein, represses flowering by transcriptional activation of Arabidopsis FLOWERING LOCUS C. Plant Cell 18(11):2985-2998

Kumar P, Anaya J, Mudunuri SB, Dutta A (2014) Meta-analysis of tRNA derived RNA fragments reveals that they are evolutionarily conserved and associate with AGO proteins to recognize specific RNA targets. BMC Biol 12:1-14

Lai Z, Schluttenhofer CM, Bhide K, Shreve J, Thimmapuram J, Lee SY, Yun D-J, Mengiste T (2014) MED18 interaction with distinct transcription factors regulates multiple plant functions. Nat Commun 5:3064. doi:10.1038/ncomms4064

Lee YS, Shibata Y, Malhotra A, Dutta A (2009) A novel class of small RNAs: tRNA-derived RNA fragments (tRFs). Genes Dev 23:2639-2649. doi:10.1101/gad.1837609

Li CF, Henderson IR, Song L, Fedoroff N, Lagrange T, Jacobsen SE (2008) Dynamic regulation of ARGONAUTE4 within multiple 
nuclear bodies in Arabidopsis thaliana. PLoS Genet 4(2):e27. doi:10.1371/journal.pgen.0040027

Li Z, Ender C, Meister G, Moore PS, Chang Y, John B (2012) Extensive terminal and asymmetric processing of small RNAs from rRNAs, snoRNAs, snRNAs, and tRNAs. Nucleic Acids Res 40:6787-6799. doi:10.1093/nar/gks307

Li Q, Hu B, Hu G, Chen C, Niu X, Liu J, Zhou S, Zhang C, Wang Y, Deng Z-F (2016) tRNA-derived small non-coding RNAs in response to Ischemia Inhibit Angiogenesis. Sci Rep 6:20850. doi:10.1038/srep20850

Livak KJ, Schmittgen TD (2001) Analysis of relative gene expression data using real-time quantitative PCR and the $2(-\Delta \Delta C(T))$ Method. Methods 25:402-408. doi:10.1006/meth.2001.1262

Loss-Morais G, Waterhouse PM, Margis R (2013) Description of plant tRNA-derived RNA fragments (tRFs) associated with argonaute and identification of their putative targets. Biol Direct 8:6. doi:10.1186/1745-6150-8-6

MacIntosh GC, Hillwig MS, Meyer A, Flagel L (2010) RNase T2 genes from rice and the evolution of secretory ribonucleases in plants. Mol Genet Genomics 283:381-396. doi:10.1007/ s00438-010-0524-9

Megel C, Cognat V, Morelle GR, Ubrig E, Molinier J, Duchêne AM, Drouard L (2015) Arabidopsis tRNA-derived RNA Fragments as a new source of small noncoding RNAs. Poster session presented at: the 26th International Conference on Arabidopsis Research; July 5-9; Paris, France

Mei Y, Yong J, Liu H, Shi Y, Meinkoth J, Dreyfuss G, Yang X (2010) tRNA binds to cytochrome $\mathrm{c}$ and inhibits caspase activation. Mol Cell 37:668-678. doi:10.1016/j.molcel.2010.01.023

Mi S, Cai T, Hu Y, Chen Y, Hodges E, Ni F, Wu L, Li S, Zhou H, Long C, Chen S, Hannon GJ, Qi Y (2008) Sorting of small RNAs into Arabidopsis Argonaute complexes is directed by the 50 terminal nucleotide. Cell 116-127. doi:10.1016/j. cell.2008.02.034

Moore B, Zhou L, Rolland F, Hall Q, Cheng W-H, Liu Y-X, Hwang I, Jones T, Sheen J (2003) Role of the Arabidopsis glucose sensor HXK1 in nutrient, light, and hormonal signaling. Science 300:332-336. doi:10.1126/science.1080585

Nogueira FTS, Sarkar AK, Chitwood DH, Timmermans MCP (2006) Organ polarity in plants Is specified through the opposing activity of two distinct small regulatory RNAs. Cold Spring Harb Symp Quant Biol 71:157-164. doi:10.1101/sqb.2006.71.045

Olovnikov I, Chan K, Sachidanandam R, Newman D, Aravin A (2013) Bacterial Argonaute samples the Transcriptome to identify Foreign DNA. Mol Cell 51:594-605. doi:10.1016/j.molcel.2013.08.014

Olvedy M, Scaravilli M, Hoogstrate Y, Visakorpi T, Jenster G, MartensUzunova ES (2016) A comprehensive repertoire of tRNA-derived fragments in prostate cancer. Oncotarget. 7(17):24766-24777. doi:10.18632/oncotarget.8293.

Ortiz-Morea FA, Vicentini R, Silva GFF, Silva EM, Carrer H, Rodrigues AP, Nogueira FTS (2013) Global analysis of the sugarcane microtranscriptome reveals a unique composition of small RNAs associated with axillary bud outgrowth. J Exp Bot 64:2307-2320. doi:10.1093/jxb/ert089

Parisien M, Wang X, Pan T (2013) Diversity of human tRNA genes from the 1000-genomes project. RNA Biol 10:1853-1867. doi: $10.4161 /$ rna.27361

Phizicky EM, Hopper AK (2010) tRNA biology charges to the front. Genes Dev 24:1832-1860

PriggeMJ,BezanillaM(2010)Evolutionarycrossroadsindevelopmental biology: Physcomitrella patens. Development137(21):3535-3543

Raina M, Ibba M (2014) tRNAs as regulators of biological processes. Front Genet 5:171. doi:10.3389/fgene.2014.00171
Rook F, Hadingham SA, Li Y, Bevan MW (2006) Sugar and ABA response pathways and the control of gene expression. Plant Cell Environ 29:426-434. doi:10.1111/j.1365-3040.2005.01477.x

Rose NR, Klose RJ (2014) Understanding the relationship between DNA methylation and histone lysine methylation. Biochim Biophys Acta-Gene Regul Mech 1839:1362-1372.

Smeets K, Opdenakker K, Remans T, Van Sanden S, Van Belleghem F, Semane B, Horemans N, Guisez Y, Vangronsveld J, Cuypers A (2009) Oxidative stress-related responses at transcriptional and enzymatic levels after exposure to $\mathrm{Cd}$ or $\mathrm{Cu}$ in a multipollution context. J Plant Physiol 166:1982-1992. doi:10.1016/j. jplph.2009.06.014

Soares AR, Fernandes N, Reverendo M, Araújo HR, Oliveira JL, Moura GM, Santos MA (2015) Conserved and highly expressed tRNA derived fragments in zebrafish. BMC Mol Biol 16:22. doi:10.1186/s12867-015-0050-8

Sobala A, Hutvagner G (2013) Small RNAs derived from the $5^{\prime}$ end of tRNA can inhibit protein translation in human cells. RNA Biol 10:553-563. doi:10.4161/rna.24285

Tamura K, Peterson D, Peterson N, Stecher G, Nei M, Kumar S (2011) MEGA5: molecular evolutionary genetics analysis using maximum likelihood, evolutionary distance, and maximum parsimony methods. Mol Biol Evol 28:2731-2739. doi:10.1093/molbev/ msr121

Thompson DM, Lu C, Green PJ, Parker R (2008) tRNA cleavage is a conserved response to oxidative stress in eukaryotes. RNA 14:2095-2103. doi:10.1261/rna.1232808

Tuck AC, Tollervey D (2011) RNA in pieces. Trends Genet 27:422432. doi:10.1016/j.tig.2011.06.001

Varkonyi-Gasic E, Wu R, Wood M, Walton EF, Hellens RP (2007) Protocol: a highly sensitive RT-PCR method for detection and quantification of microRNAs. Plant Methods 3:12

Vazquez F, Gasciolli V, Crété P, Vaucheret H (2004) The nuclear dsRNA binding protein HYL1 is required for microRNA accumulation and plant development, but not posttranscriptional transgene silencing. Curr Biol 14:346-351. doi:10.1016/j.cub.2004.01.035

Xie Z, Johansen LK, Gustafson AM, Kasschau KD, Lellis AD, Zilberman D, Jacobsen SE, Carrington JC (2004) Genetic and functional diversification of small RNA pathways in plants. PLoS Biol 2:E104. doi:10.1371/journal.pbio.0020104

Xie Z, Allen E, Wilken A, Carrington JC (2005) DICER-LIKE 4 functions in trans-acting small interfering RNA biogenesis and vegetative phase change in Arabidopsis thaliana. Proc Natl Acad Sci USA 102:12984-12989. doi:10.1073/pnas.0506426102

Xiong Y, Steitz TA (2006) A story with a good ending: tRNA 3'-end maturation by CCA-adding enzymes. Curr Opin Struct Biol 16:12-17. doi:10.1016/j.sbi.2005.12.001

Yamasaki S, Ivanov P, Hu G-F, Anderson P (2009) Angiogenin cleaves tRNA and promotes stress-induced translational repression. J Cell Biol 185:35-42. doi:10.1083/jcb.200811106

Ye R, Chen Z, Lian B, Rowley MJ, Xia N, Chai J, Li Y, He X-J, Wierzbicki AT, Qi Y (2015) A dicer-independent route for biogenesis of siRNAs that direct DNA Methylation in Arabidopsis. Mol Cell 61:1-14. doi:10.1016/j.molcel.2015.11.015

Yeung ML, Bennasser Y, Watashi K, Le SY, Houzet L, Jeang KT (2009) Pyrosequencing of small non-coding RNAs in HIV-1 infected cells: evidence for the processing of a viral-cellular double-stranded RNA hybrid. Nucleic Acids Res 37:6575-6586. doi:10.1093/nar/gkp707

Zhang J-F, Yuan L-J, Shao Y, Du W, Yan D-W, Lu Y-T (2008) The disturbance of small RNA pathways enhanced abscisic acid response and multiple stress responses in Arabidopsis. Plant Cell Environ 31:562-574. doi:10.1111/j.1365-3040.2008.01786.x 